\title{
Cataract after exposure to non-ionizing radiant energy
}

\author{
MILTON M. ZARET, WENDY Z. SNYDER, AND LEO BIRENBAUM \\ From New York University Medical Center, Departments of Ophthalmology and Physiology, and \\ Polytechnic Institute of New York, Department of Electrical Engineering and Electrophysics
}

The case reports that follow suggest that infrared radiation emitted from an electric oven and stove could be a contributory or aetiological factor in the differential diagnosis of cataracts.

During the past 30 years, there has been a constant increase in the number of devices such as diathermy machines, microwave ovens, and electric stoves which emit radiant energy. While industrial heat (infrared) cataract in glassblowers and furnace workers was first recognized nearly 200 years ago (Wenzel, 1786), lens damage caused by microwave irradiation is a disease related to modern technology (Daily, Wakim, Herrick, Parkhill, and Benedict, 1952; Richardson, Lomax, Nichols, and Green, 1952; Shimkovich and Shilyaev, 1959; Zaret, 1964; Bouchat and Marsol, 1967; Kurz and Einaugler, 1968; Zaret, 1974a, b). In both types of cataract the aetiology of the lesion has usually been explained in terms of thermal damage from absorbed radiation. In view of the long latent period often observed between exposure to repeated, subliminal doses of infrared or microwave radiation and the appearance of clinical cataracts, it has been suggested (DukeElder, 1972a) that single exposures to such radiations, which are not of themselves harmful, may become hazardous if repeated sufficiently often. Furthermore, the shorter the interval between subthreshold irradiations, the more likely it becomes that such lens changes ultimately will develop.

In this paper we present two case reports, that of a wife and husband, with different histories of exposure to non-ionizing radiant energy. Each individual developed a specific type of ocular lesion relatable to exposure. The woman, who received medical diathermy treatment for a back injury and, in later years, cooked on an electric oven and range, developed capsular cataracts in both eyes; the delayed type of cataractogenesis after diathermy therapy, instead of remaining latent, appeared to be exacerbated and hastened by additional exposure to infrared emission from the cooking device. Her husband, who had a longer history of exposure to the electric range only, developed first a retinopathy and later a lens capsule opacity both resembling injury from non-ionizing radiation. Clinical and

Address for reprints: Milton M. Zaret, MD, 1230 Post Road, Scarsdale, New York 10583, USA histological evaluation of the cataractogenesis and capsulopathy will be discussed.

\section{Case reports}

CASE I

This 46-year-old White woman was first examined on 4 January 1975, having been referred because of unusual cataracts. During the previous two years, her eyeglasses had been changed at approximately threemonthly intervals because of changes in refractive error and an intermittent esotropia. Visual acuity was correctable to $20 / 20$ minus for the right eye and $20 / 30$ minus for the left eye; her only complaint regarding clarity of vision was that it appeared as if she were looking through a transparent curtain with her left eye.

Slit-lamp examination revealed a large, irregularly outlined, honeycombed capsulopathy involving more than half of the posterior surface of the lens. A few regions of vesiculation and opacification were present in the lens substance at its interface with the capsule. Thus, all of the disease was limited to the posterior lens capsule and adjacent, contiguous surfaces of the lens substance. This form of capsular cataract was present bilaterally, being slightly more extensive in the left than the right lens. Fig. I is a photograph of the left eye taken at the time of this examination.

There was nothing in the patient's history to suggest an aetiology except exposure to a course of medical diathermy as therapy for back pain approximately 15 years earlier.

The diagnosis was incipient, delayed radiant energy cataract. The patient was advised that this type of cataract ordinarily would lie dormant or progress very slowly over a time period measured in years, that no treatment other than surgery was available, that surgery was not indicated at this time, and that she should avoid any additional exposure to radiant energy, especially nonionizing radiation such as from medical diathermy or microwave ovens. She was instructed to return for a follow-up examination after an interval of three to six months because no significant changes were expected to occur sooner.

However, within two months the patient had observed both a further decrease in vision which now made driving at night hazardous and increased difficulty in reading because the esotropia recurred more frequently. Therefore, she returned for re-examination on 15 March. At this time the best correctable visual acuity of the left eye was reduced to $20 / 40$ minus due to progression of the cataractous changes. It was also noted that after 


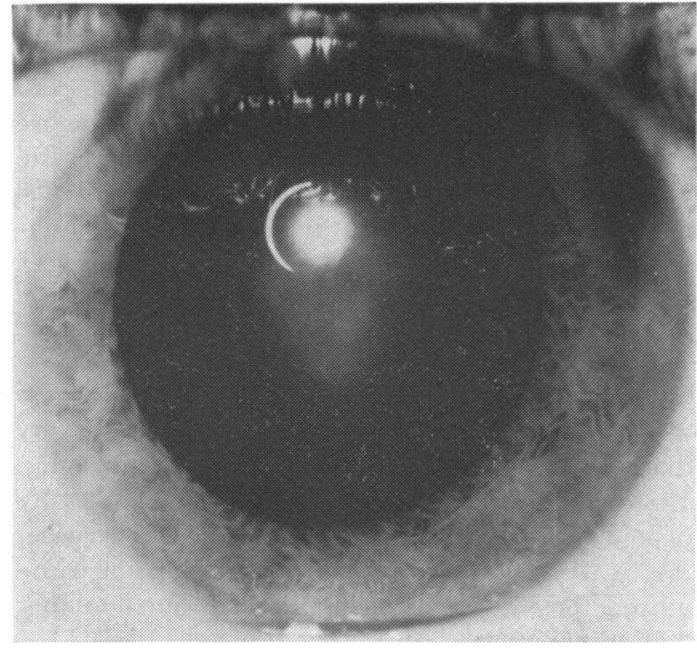

FIG. I Lens photcgraph, Case I, left eye. Note cataract formation limited to posterior surface of lens

instillation of mydriatics the pupils did not dilate well; this is not an uncommon, late finding in non-ionizing radiation cataracts, occasionally indicating the formation of posterior synechiae located peripherally, at the point where the iris first lies in apposition with the anterior bulge of the lens. This suspicion of synechiae was confirmed at cataract surgery performed on 15 April, when it was necessary to perform posterior synechiotomies to free the lens from iridial adhesions in order to extract the cataract without rupturing the lens capsule.

By 9 August 1975 , the cataractous process in the right eye had also become more extensive, the lesion now involved most of the posterior surface of the lens and, in addition, a marked capsulopathy had become evident at its anterior surface. The patient's complaints were hazy vision and intermittent diplopia. Visual acuity was reduced to $20 / 50$ plus or minus.

Cataract extraction was performed on the right eye on 20 August. At surgery, the anterior capsule was found to be adherent to the iris and the posterior capsule was adherent to the face of the vitreous. The adhesions were lysed and the lens was then delivered from the eye intracapsularly. After surgery, visual acuity was correctable to $20 / 20$ plus in each eye separately and the resultant restoration of the ability for fusion corrected the intermittent esotropia.

In view of the unusually rapid development of this patient's cataracts, which took place over a period of months rather than years, an investigation was undertaken to determine whether some additional radiant energy source in the patient's environment could have been providing repeated, covert exposures, thus being a contributory factor. The only suspicious element was that, since her marriage on 8 December 1973, she had used an electric range and oven. Both she and her husband worked and, as he returned home earlier than she, frequently he would cook the dinner. For this reason, it was decided to examine his eyes for signs of radiant energy effects.

\section{CASE 2}

This asymptomatic 55-year-old man was first examined on 3 May 1975. His medical and occupational histories were negative regarding serious illness or significant exposure to non-ionizing radiation.

The examination findings were physiological except for ophthalmoscopy. In the left eye, the macula exhibited pigmentary changes, generalized hyperpigmentation with a few scattered minute areas of depigmentation, a finding seen rarely in general population groups but not infrequently in workers where the occupational environment has an increased ambiance of photic energy (infrared and high intensity light environments) (DukeElder, 1972b; Zaret, 1972). Also in the left eye, a small area of pigmentary, degenerative change was present superiorly between the macula and optic disc. These changes are shown in Fig. 2, a retinal photograph of the left eye. In the right eye, two minute areas of depigmentation, each about $0.1 \mathrm{~mm}$ in diameter, were found in the inferior region of the macula. By slit-lamp examination, both lenses were entirely physiological.

After an interval of six months, the patient was reexamined on 22 November 1975. His visual acuity had remained correctable to $20 / 20$ in each eye. However, the maculopathy had become more marked in both eyes. More importantly, by slit-lamp biomicroscopy a small area of capsular opacification measuring about $\mathrm{I} \cdot 0 \mathrm{~mm}$ in largest diameter had now become apparent at the posterior surface of the left lens. These retinal and lens findings are compatible with frequently repeated exposure to non-ionizing radiation.

In view of the implication that the electric oven and range could have served as a harmful, covert source of radiant energy exposure for both patients, being contributory to Case I and causal for Case 2, measurements were made of its emissions.

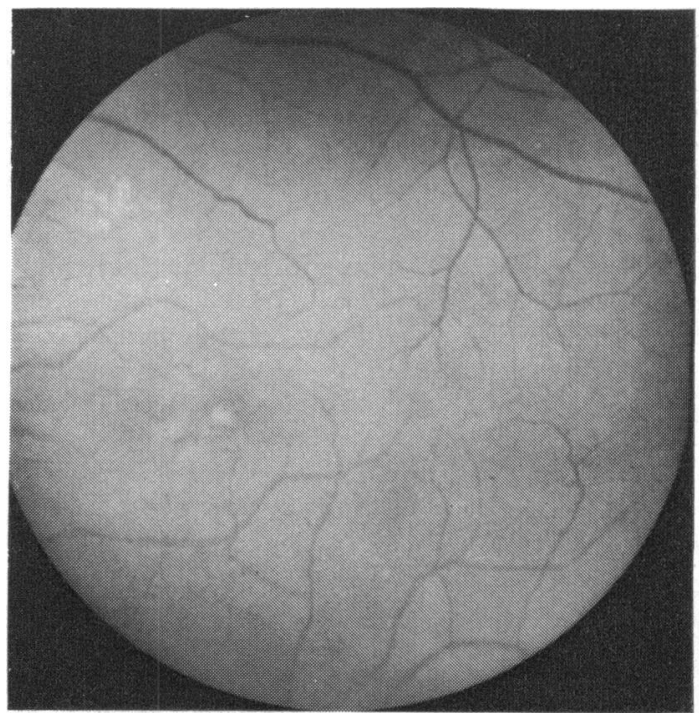

FIG. 2 Retinal photograph, Case 2, left eye. Note pigmentary degenerative changes 


\section{MEASUREMENTS OF ELECTRIC OVEN AND RANGE EMISSION}

Measurement of the radiant energy emission was accomplished by using a calibrated Eppley thermopile and a Keithley model $150 B$ microvolt ammeter.

Irradiation measurements of the electric oven with the controls set at the 'broil' position were taken at a distance of $30.4 \mathrm{~cm}$ in front of the door. The values were $24 \mathrm{~mW} / \mathrm{cm}^{2}$ with the door closed and $97 \mathrm{~mW} / \mathrm{cm}^{2}$ with the door open.

The electric range had four burners, two in front and two at the back. Measurements were taken at a distance of $45.7 \mathrm{~cm}$ in front of the range. With one front burner on 'high', the irradiation flux was $57 \mathrm{~mW} / \mathrm{cm}^{2}$. With one front and the ipsilateral rear burner on 'high', the measured emission was $97 \mathrm{~mW} / \mathrm{cm}^{2}$. No measurements were made with all of the stove sources emitting, as such a flux of radiant energy would have exceeded by far the solar constant, an obviously hazardous condition for direct exposure of the eyes.

\section{Results}

\section{HISTOLOGICAL FINDINGS}

After cataract extraction the right lens was immediately placed in 4 per cent glutaraldehyde buffered with $0.1 \mathrm{~mol} / 1$ sodium cacocdylate for 120 hours at room temperature (approximately $24^{\circ} \mathrm{C}$ ). The tissue was rinsed in buffer with 4 per cent sucrose, sliced into anterior and posterior segments and postfixed for four hours in I per cent osmium tetroxide buffered with the cacocdylate-sucrose medium. After further rinsing in buffer and dehydration in a graded series of alcohols, the tissue was trimmed and cut into small strips and embedded in epon. Thick ( 1 to $2 \mu \mathrm{m}$ ) sections were cut on a Porter-Blum (M-2) ultra-microtome, stained with I per cent methylene blue and photographed under oil $(\times 560)$ with a Zeiss photomicroscope.

Fig. 3 shows the anterior surface of the lens lying behind the iris. Note the posterior synechiae with pigmented precipitates deposited on the anterior lens capsule. The subcapsular epithelium appears vacuolated and several cell nuclei seem pyknotic. In Fig. 4 a more central area of the anterior surface of the lens is shown. Here, the nonuniform staining of the capsule is apparent and the subcapsular epithelium is vacuolated.

Figs 5 and 6 represent the posterior surface of the lens. In Fig. 5 note the irregular, outer surface of the posterior capsule and the vacuolization of the posterior cortex. The splitting of the capsule from the subcapsular cortex is an artefact. Fig. 6 shows the non-uniform staining of the posterior capsule. There appears to be a large area of subcapsular degeneration of the cortex as well as vacuolization of discrete areas. A close inspection of the inner surface of the capsule reveals that lens substance is adherent to it, thus obliterating the potential subcapsular space. Another feature of this apparent adhesion of the lens substance to capsule is that the lens substance at this site is more densely stained. Perhaps this may explain the earliest clinical appearance by slit-lamp biomicroscopy of capsulopathy in non-ionizing radiation cataract.

\section{Discussion}

The clinical findings and course for Case I suggest that the earlier exposure to medical diathermy radiation produced a covert, slowly progressing, pathological process which was later accelerated by repeated exposure to infrared radiation. Otherwise, there was an unusually short duration of time required for the full evolution of the non-ionizing radiation cataracts.

The histological appearance of the cataractous lens includes features previously described in experimental and clinical lesions produced after repeated, subliminal exposure to infrared or microwave irradiation. The pathological findings of the anterior surface of the lens-including pigment deposition on the anterior capsule, swelling and vacuolization of the subcapsular epithelium, and neighbouring lens fibres in association with posterior subcapsular vacuolization-are typical of of an infrared lesion (Vogt, r930; Goldmann, 1932; Langley, Mortimer, and McCulloch, I960). Vesiculation or honeycomb appearance at the posterior capsule and subcapsular cortex is more characteristic of medical diathermy or microwave-induced cataracts (Zaret, I964; Bouchat and Marsol, I967; Kurz and Einaugler, 1968; Zaret, 1974a and b). This patient exhibited both infrared and diathermy radiation findings. Thus it appears that the histological evaluation of the lens supports the clinical impression that the more recent infrared exposure was effective quickly because of earlier exposure from medical diathermy.

The pathogenic cause of non-ionizing radiant energy cataract is probably not as simple as some investigators have implied. For example, infrared rays are reported to be absorbed mainly by the pigment epithelium of the iris (Goldmann, 1932; Langley and others, 1960), and converted to heat which may damage the adjacent capsule and subcapsular epithelium of the lens. Local increase in temperature at the anterior surface of the lens, normally $1.5{ }^{\circ} \mathrm{C}$ cooler than the posterior pole, can be produced experimentally with infrared radiation (Goldmann, 1932). However, the delayed-type of microwave cataract usually is observed at the posterior surface of the lens and can be produced experimentally more easily in albino than in pigmented rabbits (Daily and others, 1952). Here, 


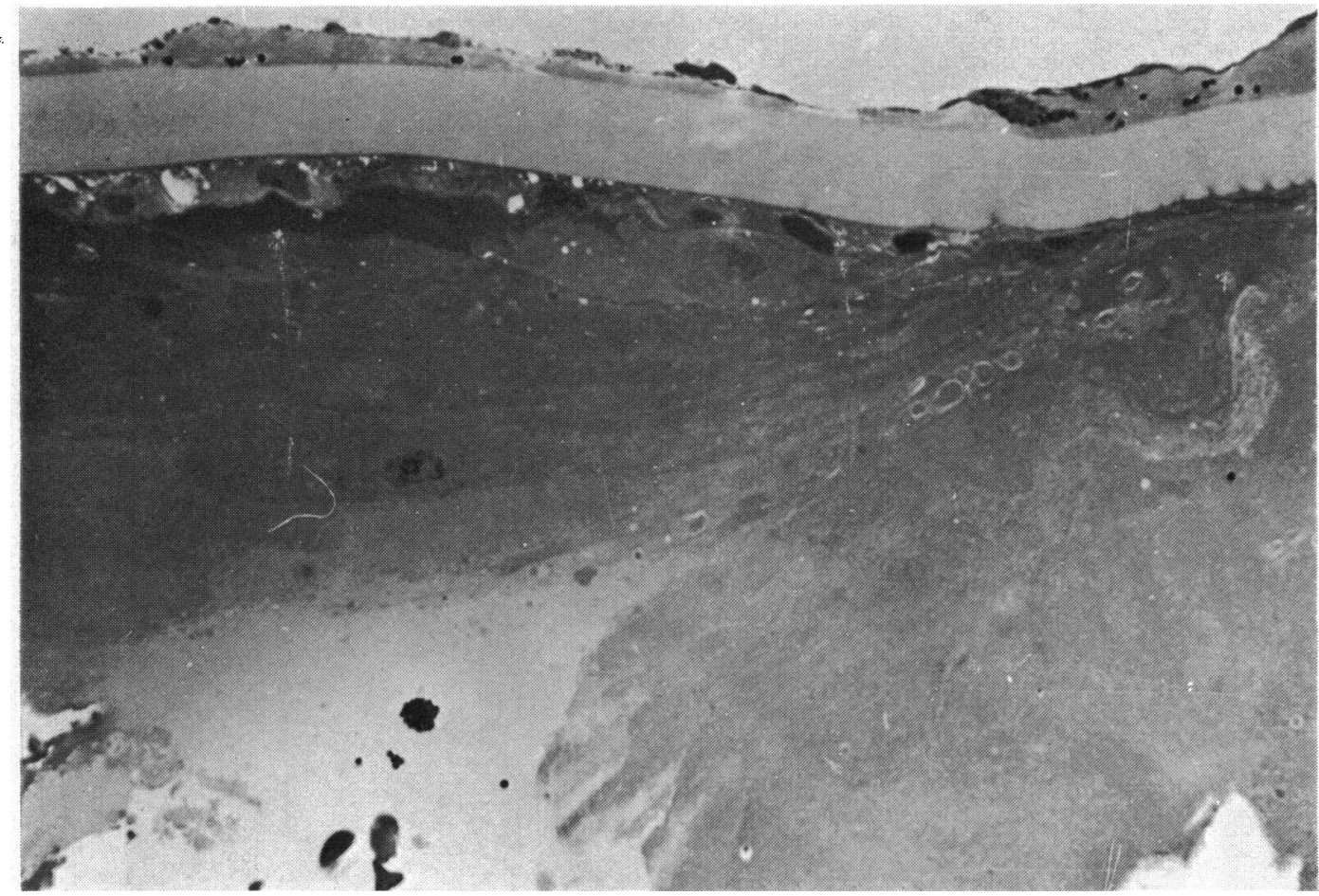

FIG. 3 Anterior surface of lens, behind the iris (methylene blue, $\times 560$ ). Note pigmented precipitates on lens capsule and vacuolization of subcapsular epithelium

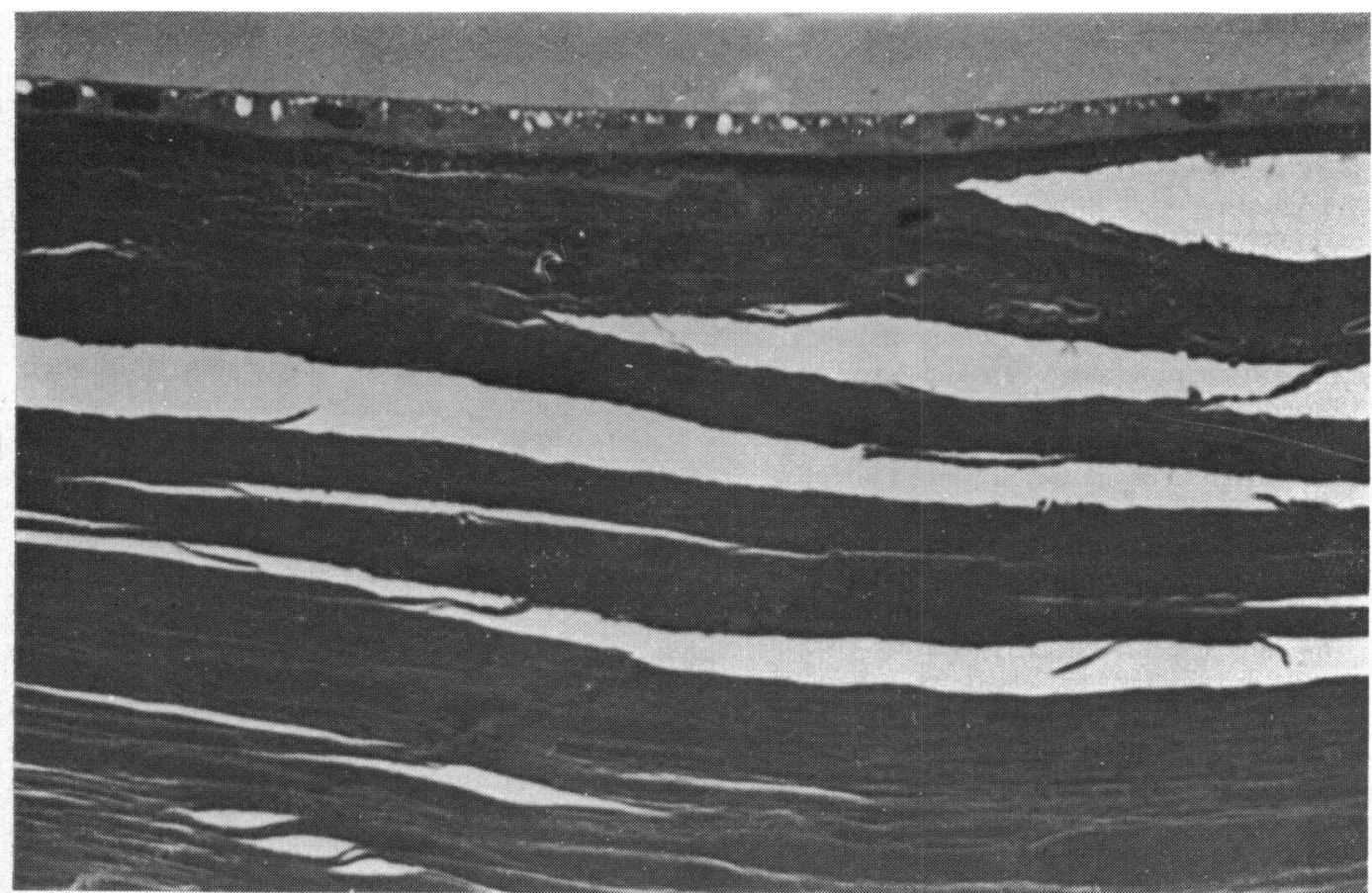

FIG. 4 Anterior surface of lens, near pupillary aperture (methylene blue, $\times 560$ ). Note banded staining of the capsule and vacuolization of subcapsular epithelium 


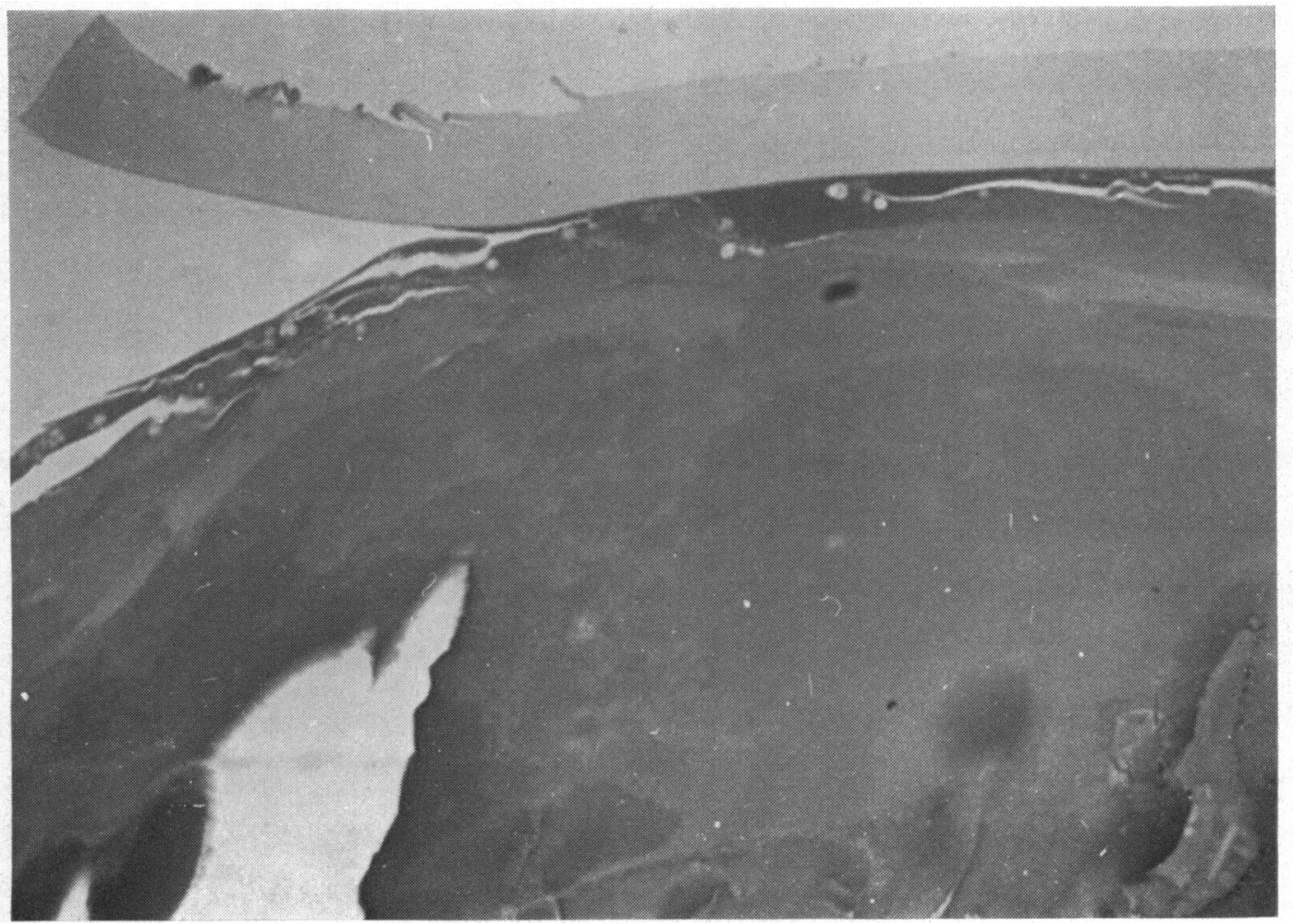

FIG. 5 Posterior surface of lens (methylene blue, $\times$ 560). Note irregular, outer capsule surface, and vacuolization of subcapsular cortex

a direct heat effect from absorption by the pigment of the iris seems unlikely.

There is a growing body of scientific data (Czerski, 1974) demonstrating that long-term, chronic, or frequently repeated exposure to low field intensities of non-ionizing radiation can produce non-thermal effects on man. These are not associated with recognizable signs or symptoms of whole or partial increases in body temperature. In the lens, the clinical observation of a long latent period measured in months or years between exposure and effect suggests that these lesions are not produced directly by a local increase in temperature during irradiation of the tissue. In addition, the adhesions between iris and lens capsule also develop later. These findings suggest that perhaps the radiant energy damages the lens capsule directly (Zaret, 1972), altering its permeability or elasticity, thereby disrupting normal protein metabolism in the adjacent lens fibres. The injured capsule and subsequent permeation of proteolytic products through it may result in a proteopexic adhesion to whatever adjacent tissues lie in contact with the lens. Such a process could explain the clinical findings of adhesions of the lens capsule not only to the iris but also to the face of the vitreous and thus provide a rationale for the delayed development of these adhesions.

In Case 2, the maculopathy is indistinguishable from that seen in radiational chorioretinitis after repeated exposure to infrared radiation over an extended period of time. However, it is more important to note that capsular opacification has just become evident in the left lens, indicating that cataractogenesis is at a very early stage. Although one would anticipate a slow progression of the radiational injuries should the exposures continue, as a preventive measure the patient was advised to discontinue unnecessary, further exposure to any intense source of radiant energy, including the electric oven and stove.

\section{Summary}

The case histories of two individuals exposed to thermal radiation emitted from an electric oven and range were presented. In one patient, earlier exposure to medical diathermy appears to have initiated delayed or late-appearing, capsular cataracts. Instead of the anticipated slow progression, the cataractogenesis was accelerated following recent, repeated exposure to the intense, infrared 


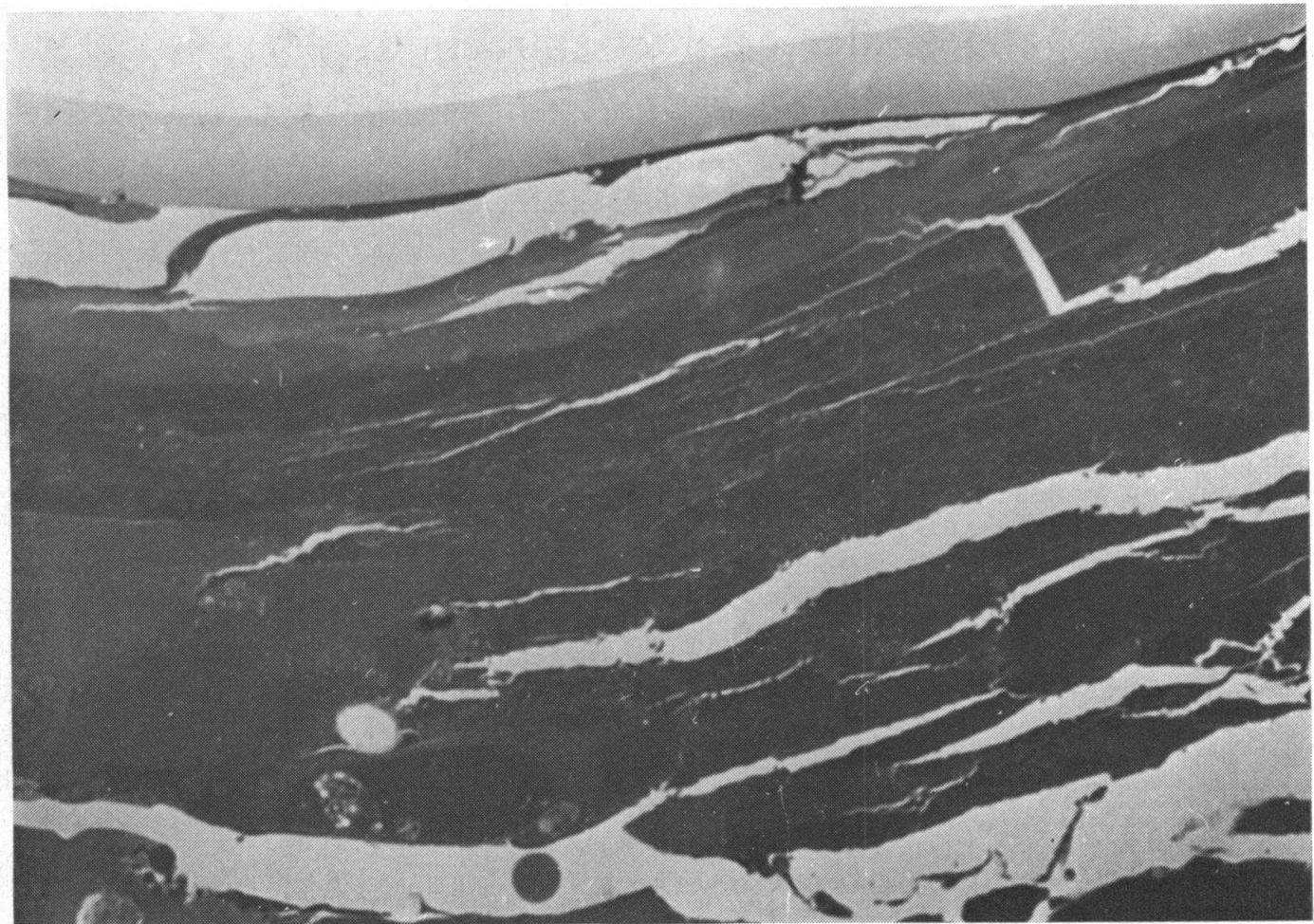

FIG. 6 Posterior surface of lens (methylene blue, $\times 560$ ). Note banded staining of capsule and degenerated areas of subcapsular cortex

radiation. In the other patient, exposed solely to infrared radiation, a chorioretinal lesion indistinguishable from the type characteristically secondary to repeated, thermal radiation was observed. More recently, the earliest sign of thermal radiation cataractogenesis, capsular opacification, has become evident.
The widespread availability of radiant energy sources such as diathermy machines, microwave ovens, and electric ovens and ranges makes it imperative to examine carefully any possible hazards that may result from their use. With this in mind, radiant energy should be considered in the differential diagnosis of cataractogenesis.

\section{References}

вouchat, J., and marsol, c. (1967) Arch. Ophtal. (Paris), 27, 593

CZERSKI, P., ed. (1974) 'Biological Effects and Health Hazards of Microwave Radiation, Proceedings of an

International Symposium'. Polish Medical Publishers, Warsaw

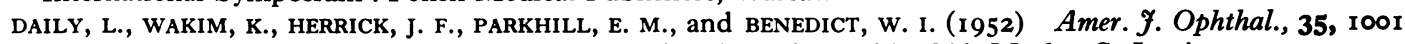
DUKE-ELDER, S. (1972a) 'System of Ophthalmology', vol. XIV, pp. 865-866. Mosby, St Louis

(1972b) Ibid., pp. 888-894

goldmann, H. (1932) Arch. Ophthal., 128, 648

KURz, G. H., and EINAUGLER, R. B. (1968) Amer. F. Ophthal., 66, 866

LANGLEY, R. K., MORTIMER, M. B., and MCCUlloch, C. (1960) Arch. Ophthal., 63, I 7

richardson, A. W., LomaX, D. H., Nichols, J., and GREen, H. D. (1952) Amer. Э. Ophthal., 35, 993

SHIMkovich, I. S., and Shilyaev, v. G. (1959) Vestn. Oftal., 72, 12

vogt, A. (1930) Klin. Mbl. Augenheilk., 85, 321

WENZEL, M. DE (1786) Cited by S. Duke-Elder, in 'System of Ophthalmology', vol. XIV, p. 878. Mosby, St Louis zARET, M. M. (1964) Technical Documentary Report No. RADC-TDR-64-273, Cont. AF 30 (602)-3097, p. 13.

Griffiss Air Force Base, Rome, New York

$$
\text { (1972) IEEE Trans. biomed. Eng., BME-19, } 313
$$

(1974a) N.Y. St. Y. Med., 74, 2032

(1974b) 'Biologic Effects and Health Hazards of Microwave Radiation, Proceedings of an International

Symposium', ed. P. Czerski. Polish Medical Publishers, Warsaw 